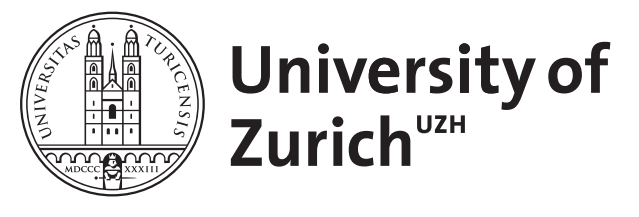

\title{
Declarative serializable snapshot isolation
}

Tilgner, Christian ; Glavic, Boris ; Böhlen, Michael ; Kanne, Carl-Christian

\begin{abstract}
Snapshot isolation (SI) is a popular concurrency control protocol, but it permits non-serializable schedules that violate database integrity. The Serializable Snapshot Isolation (SSI) protocol ensures (view) serializability by preventing pivot structures in SI schedules. In this paper, we leverage the SSI approach and develop the Declarative Serializable Snapshot Isolation (DSSI) protocol, an SI protocol that guarantees serializable schedules. Our approach requires no analysis of application programs or changes to the underlying DBMS. We present an implementation and prove that it ensures serializability.
\end{abstract}

DOI: https://doi.org/10.1007/978-3-642-23737-9_13

Posted at the Zurich Open Repository and Archive, University of Zurich

ZORA URL: https://doi.org/10.5167/uzh-56401

Conference or Workshop Item

Accepted Version

Originally published at:

Tilgner, Christian; Glavic, Boris; Böhlen, Michael; Kanne, Carl-Christian (2011). Declarative serializable snapshot isolation. In: 15th International Conference in Advances in Databases and Information Systems, Vienna. Austria, 19 September 2011 - 24 September 2011. Springer, 170-184.

DOI: https://doi.org/10.1007/978-3-642-23737-9_13 


\title{
Declarative Serializable Snapshot Isolation
}

\author{
Christian Tilgner $^{1}$, Boris Glavic ${ }^{2}$, Michael Böhlen ${ }^{3}$, Carl-Christian Kanne ${ }^{4}$ \\ ${ }^{1,3}$ University of Zurich, ${ }^{2}$ University of Toronto, ${ }^{4}$ University of Mannheim \\ ${ }^{1}$ tilgner@ifi.uzh.ch, ${ }^{2}$ glavic@cs.toronto.edu ${ }^{3}$ boehlen@ifi.uzh.ch, \\ ${ }^{4}$ kanne@informatik.uni-mannheim.de
}

\begin{abstract}
Snapshot isolation (SI) is a popular concurrency control protocol, but it permits non-serializable schedules that violate database integrity. The Serializable Snapshot Isolation (SSI) protocol ensures (view) serializability by preventing pivot structures in SI schedules. In this paper, we leverage the SSI approach and develop the Declarative Serializable Snapshot Isolation (DSSI) protocol, an SI protocol that guarantees serializable schedules. Our approach requires no analysis of application programs or changes to the underlying DBMS. We present an implementation and prove that it ensures serializability.
\end{abstract}

\section{Introduction}

Snapshot Isolation (SI) [3] is a popular multiversion concurrency control (MVCC) protocol, but it permits non-serializable schedules. Fekete et al. [9] showed that every non-serializable SI schedule necessarily contains an access pattern with two consecutive vulnerable edges (see Sec. 2.2), to which we refer to as a pivot structure. Cahill et al. [5] presented the Serializable Snapshot Isolation (SSI) protocol that ensures serializable schedules by preventing pivot structures.

We leverage the ideas of SSI and propose the Declarative Serializable Snapshot Isolation (DSSI) protocol, a declarative technique that guarantees serializable schedules while maintaining the advantages of SI. DSSI requires no manual transaction program analysis and no modifications to application programs resp. to the underlying DBMS and is applicable to ad-hoc transactions. We implement DSSI using our declarative scheduling model called Oshiya. Oshiya models the scheduler state (including the generated schedule) in so-called scheduling relations and formalizes a protocol as a protocol specification. A protocol specification is a set of constraints specified as boolean domain relational calculus expressions that have to hold for all scheduling relation states. In Oshiya, a protocol specification is implemented as declarative scheduling queries. Request scheduling is performed by applying a generic scheduling algorithm that repeatedly executes the scheduling queries over the scheduling relations. The queries determine which of the pending requests can be added to the relation modelling the schedule without violating the protocol specification. We show how to detect and prevent pivot structures using Oshiya and implement the DSSI protocol specification as scheduling queries. This implementation is concise and close to the formal protocol specification which enables us to prove its correctness. The main contributions of the paper are: 
- We introduce DSSI, a protocol that ensures serializable SI executions, and formalize it as an Oshiya protocol specification.

- Using Oshiya we develop an SQL implementation of DSSI.

- We prove that the implementation ensures serializable schedules.

The paper structure is as follows: Sec. 2 describes SI and reviews the approach applied by the SSI protocol to detect non-serializable schedules. Sec. 3 introduces Oshiya. Sec. 4 shows how we model data snapshots and presents schemata for the scheduling relations. Sec. 5 formalizes the DSSI protocol. Sec. 6 presents the DSSI scheduler implementation. Sec. 7 proves that our implementation ensures serializable executions. Sec. 8 reviews related work and Sec. 9 concludes.

\section{Background: Snapshot Isolation and Serializability}

We model a transaction $t_{i}$ as a sequence of read and write requests (denoted as $r_{i}(x)$ resp. $w_{i}(x)$ where $x$ stands for the accessed data item). Each transaction finishes with an abort $\left(a_{i}\right)$ or commit $\left(c_{i}\right)$ request. The write-set $W S_{i}$ of $t_{i}$ contains all data items written by $t_{i}$. A history (schedule) is a sequence of interleaved executions of requests from a set of concurrent transactions. The requests in a history are totally ordered. We write $p{ }^{H} q$ if request $p$ is executed before request $q$. Let $b o t_{i}$ denote the begin of $t_{i}$ (when $t_{i}$ executed its first request) and $e^{\circ} t_{i}$ its end (when $t_{i}$ aborted resp. committed). The execution interval of a committed transaction $t_{i}$ is $\left[b o t_{i}, c_{i}\right]$, the one of a non-aborted, possibly committed transaction $t_{i}$ is $\left[b o t_{i}, l_{i}\right]\left(l_{i}\right.$ is $t_{i}$ 's latest operation). Two committed transactions $t_{i}$ and $t_{j}$ overlapped if: Overlapped ${ }_{i j} \Leftrightarrow\left[\right.$ bot $\left._{i}, c_{i}\right] \cap\left[b_{b} t_{j}, c_{j}\right] \neq \emptyset$. Two non-aborted (maybe active) transactions $t_{i}$ and $t_{j}$ overlap if: Overlap $i_{i j} \Leftrightarrow\left[b_{b} t_{i}, l_{i}\right] \cap\left[b_{b} t_{j}, l_{j}\right] \neq \emptyset$.

\subsection{Snapshot Isolation}

SI is a multiversion concurrency protocol that maintains multiple versions of data items (tuples). Each write $w_{i}(x)$ creates a new version of item $x$ that is visible to other transactions after $c_{i}$. Each read $r_{i}(x)$ accesses the latest version of $x$ written by transactions that committed before bot $_{i}$. Moreover, a transaction always sees the versions it created itself. Under SI, reads are never delayed because of write requests of concurrent transactions and vice versa. SI avoids inconsistent read anomalies because transactions never access partial results of other concurrent transactions. SI requires disjoint write-sets of concurrent committed transactions which is, e.g., ensured by the First-Committer-Wins (FCW) rule. FCW specifies that a transaction is aborted if a concurrent transaction with an overlapping write-set already committed. FCW also prevents lost updates. A typical anomaly that leads to non-serializable SI histories is the Write Skew [3], detailed in Ex. 1.

Example 1. Consider history $H_{w s}$ in Fig. 1. Initially, data items $x=50$ and $y=50$ are consistent and satisfy constraint $C=x+y \geq 0$. Transaction $t_{1}$ reads $x$ and $y$. A concurrent transaction $t_{2}$ reads $x$ and $y$, writes $x$ (after subtracting 90) and commits. Finally, $t_{1}$ writes $y$ (after subtracting 90) and commits. In the final 
state, $C$ is violated although $t_{1}$ and $t_{2}$ checked $C$ explicitly before committing. This is because $C$ is checked on the version of $x$ and $y$ that is visible to $t_{1}$ and $t_{2}$ and not on the final state resulting from their interleaved execution.

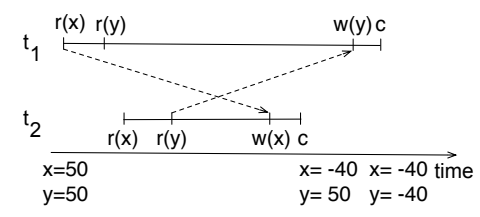

Fig. 1. History $H_{w s}$

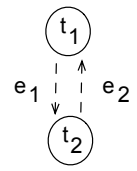

Fig. 2. MVSG for History $H_{w s}$

\subsection{Detecting Non-Serializable Histories}

Serializability of SI histories can be checked using a multiversion serialization graph $M V S G=(N, E)[5]$. The MVSG of a history $H$ is a graph that contains a node for each committed transaction $t_{i}$ of $H: t_{i} \in N \Leftrightarrow c_{i} \in H$. It contains an edge from transaction $t_{i}$ to transaction $t_{j}$ with $i \neq j$ if (a) $w_{i}(x)<^{H} w_{j}(x)$, (b) $w_{i}(x)<^{H} r_{j}(x)$ or (c) $r_{i}(x)<^{H} w_{j}(x)$. An edge of type (c) that occurs between two concurrent committed transactions $t_{i}$ and $t_{j}$ is called a vulnerable edge [9]: Overlapped ${ }_{i j} \wedge r_{i}(x)<{ }^{H} w_{j}(x)$. A pivot structure is defined as follows: Overlapped $_{i j} \wedge\left(r_{i}(x)<{ }^{H} w_{j}(x)\right) \wedge$ Overlapped $_{j k} \wedge\left(r_{j}(x)<{ }^{H} w_{k}(x)\right)$. Every MVSG of a non-serializable SI history must contain a pivot structure [9]. The existence of a pivot structure is a necessary but not sufficient condition for the non-serializability of an SI history. Thus, an SI history is serializable if its MVSG does not contain pivot structures.

Example 2. Fig. 1 shows history $H_{w s}$. Vulnerable edges are shown as dotted lines. The MVSG for $H_{w s}$ in Fig. 2 has a node for each committed transaction of $H_{w s}\left(t_{1}\right.$ and $\left.t_{2}\right)$ and two edges $e$ : $\left(e_{1}\right)$ from $t_{1}$ to $t_{2}$ due to $r_{1}(x)<^{H} w_{2}(x)$; $\left(e_{2}\right)$ from $t_{2}$ to $t_{1}$ due to $r_{2}(y)<{ }^{H} w_{1}(y) . H_{w s}$ is not serializable and, thus, the MVSG contains a pivot structure (two consecutive vulnerable edges $e_{1}$ and $e_{2}$ ).

\subsection{Serializable Snapshot Isolation Protocol}

The SSI protocol proposed by Cahill et al. [5] ensures serializability by preventing pivot structures. The main idea is to check SI histories at runtime for structures that can evolve into pivot structures. We call such structures potential pivot structures. A potential pivot structure is defined as: Overlap $p_{i j} \wedge\left(r_{i}(x)<{ }^{H}\right.$ $\left.w_{j}(x)\right) \wedge$ Overlap $_{j k} \wedge\left(r_{j}(x)<{ }^{H} w_{k}(x)\right) \wedge \neg\left(c_{i} \wedge c_{j} \wedge c_{k}\right)$. I.e., a potential pivot structure is a pivot structure without the requirement that the three (not necessarily distinct) participating transactions have committed. It evolves into a pivot structure once all participating transactions have committed. The set of transactions in potential pivot structures is naturally a superset of the transactions in pivot structures. For each detected potential pivot structure, one of the participating transactions is aborted to prevent it from evolving into a pivot structure. This approach guarantees that the resulting histories are serializable, but it may produce false positives, i.e., not every potential pivot structure finally results in a non-serializable history. Our implementation leverages this idea and aborts transactions that participate in potential pivot structures (see Sec. 6). 


\section{Declarative Scheduling Model}

We propose a declarative scheduling model [13] called Oshiya ${ }^{1}$ to model and implement DSSI. The main ideas of Oshiya are: (1) The state of a scheduler (including the history it produces) is modeled as instances of three scheduling relations: PendingRequests $(\mathcal{R})$ buffers arriving client requests for scheduling. RelevantHistory $(\mathcal{H})$ stores already executed requests in their execution order. It models the schedule generated so far. Executable $(\mathcal{E})$ buffers requests already scheduled for execution. (2) Oshiya formalizes a protocol as a set of constraints, called protocol specification, that have to hold for each generated state of $\mathcal{H}$. (3) The protocol specification constraints are implemented as declarative scheduling queries: $Q_{\text {Schedule }}, Q_{\text {Revoked }}, Q_{\text {Irrelevant }}$. Request scheduling is performed by repeatedly executing the scheduling queries over the scheduling relations to determine which of the pending requests in $\mathcal{R}$ can be added to $\mathcal{H}$ without violating the protocol specification constraints.

Example 3. For presentation purposes, we use simplified schemata for the scheduling relations in this example. Assume the following schema for relations $\mathcal{R}$ and $\mathcal{E}:(T A, O p, O b)$. For each request, $T A$ is the transaction executing the request, $O p$ is the type of operation (e.g., $r$ for a read), and $O b$ is the data object the operation accesses. Relation $\mathcal{H}$ has an additional attribute $I D$ for recording the request execution order. Using this schema, the scheduler state after scheduling the first request from history $H_{w s}$ (Fig. 1) is as follows:

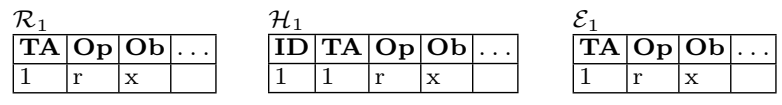

The state of the scheduler is advanced in iterative steps by applying a generic scheduling algorithm (shown on the right) that evaluates the scheduling queries over the current instances of the scheduling relations. Each iterative step (one while loop), called scheduler iteration, schedules multiple requests at once, resulting in updated instances of the scheduling relations. This is in contrast to

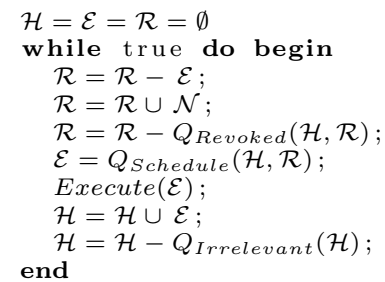

DBMSs that schedule requests individually. The algorithm is the same for every protocol, but it is parameterized by the protocol specific schema of the scheduling relations and the scheduling queries. $\mathcal{N}$ is the set of newly arrived client requests. $Q_{\text {Revoked }}$ identifies nonexecutable requests (e.g., deadlocked) (line 5). $Q_{\text {Schedule }}$, the main scheduling query, identifies which pending requests from $\mathcal{R}$ should be selected for execution in this iteration (line 6). Q Irrelevant returns requests that are irrelevant for future scheduling decisions. They are removed from $\mathcal{H}$ (line 9 ) to prevent $\mathcal{H}$ from growing infinitely. Note that in the remainder of this paper we limit the discussion to $Q_{\text {Schedule }}$.

Example 4. Reconsider the scheduler state from Ex. 3. Two new requests got inserted into $\mathcal{R}$ at the beginning of scheduler iteration 2: $r_{1}(y), r_{2}(x)$. Assume

${ }^{1}$ Oshiya refers to the passenger arrangement staff at Japanese train stations who help to fill a train by pushing people onto the train or guiding them to free railway cars. 
that running the scheduling queries selected both request from $\mathcal{R}$ for execution. This leads to the following updated scheduler state:
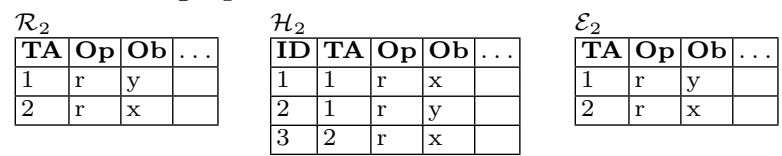

Applying the scheduling queries to a set of newly arrived requests $\mathcal{N}$, each scheduler iteration produces new instances of the scheduling relations $\mathcal{R}, \mathcal{H}$ and $\mathcal{E}$. This yields a sequence of states of $\mathcal{H}$ called history, defined below. We use this definition of history for the same purpose as traditional definitions of histories are used by other approaches: to reason over the properties of a protocol. An advantage of Oshiya is that we can use the same kind of reasoning to prove the correctness of a scheduler implementation.

Definition 1 (History). Let $\mathbb{I}=<\mathcal{N}_{0}, \ldots>$ be a sequence of sets of input requests. Let $q$ be protocol-specific versions of the scheduling queries. We define the history $\mathbb{H}_{q}(\mathbb{I})$ generated according to $q$ over input $\mathbb{I}$ as $\left\langle\mathcal{H}_{0}, \ldots\right\rangle$, where $\mathcal{H}_{i}$, called a history state, is the state of relation $\mathcal{H}$ after the $i^{\text {th }}$ scheduler iteration produced using $q$ to parameterize the generic algorithm and $\mathcal{N}_{i}$ as input $\mathcal{N}$. In the paper, we drop $q$ and $\mathbb{I}$ if it is clear from the context and solely use $\mathbb{H}$.

In the remainder of this paper, we use $\mathcal{H}$ to denote both the history relation and one history state and drop indices on $\mathcal{H}$ if the scheduler iteration is irrelevant for the discussion (same holds for $\mathcal{R}, \mathcal{E}$ and $\mathcal{N}$ ). According to the algorithm presented above, the history state $\mathcal{H}_{i}$ is a cumulative snapshot, i.e., it includes all previous history states $\mathcal{H}_{j}$ with $j<i$.

Example 5. For instance, the history states shown below could be the result of scheduling over $\mathbb{I}=<\{(1, r, x),(2, r, x)\},\{(1, r, y)\},\{(2, r, y)\}>$ :

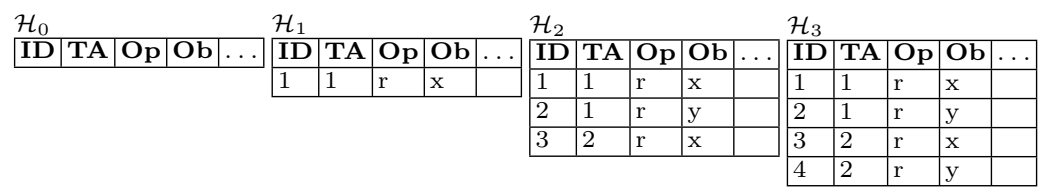

We model a protocol as a set of constraints called protocol specification. A protocol specification constraint is a boolean domain relational calculus expression over histories. We allow quantification over scheduler iterations to enable, e.g., constraints that check the order of requests in the history.

Definition 2 (Protocol Specification). A protocol specification $\Phi$ is a set of boolean domain relational calculus expressions over $\mathbb{H}$.

The formalization of a protocol as logical constraints and its implementation as queries allows us to formally reason about the correctness of an implementation. Given a protocol specification $\Phi$ and an implementation of this protocol as a set $q$ of scheduling queries, the definition presented below defines what it means for $q$ to correctly implement $\Phi$. Intuitively, this is the case if for every input $\mathbb{N}$, the history created by our scheduling algorithm using $q$ satisfies $\Phi$. We use this definition in Sec. 7 to prove the correctness of our DSSI implementation. 
Definition 3 (Correctness of Scheduling Queries). Scheduling queries $q$ satisfy a protocol specification $\Phi$, denoted as $q \models \Phi$, if for every input sequence $\mathbb{I}$ the generated history $\mathbb{H}$ produced using q satisfies $\Phi$ : $\mathbb{H}_{q}(\mathbb{I}) \models \Phi$.

\subsection{Assumptions and Notational Remarks}

We make the following assumptions: (1) Client requests read resp. manipulate only one tuple. (2) A transaction waits until its current request is executed before issuing new requests. (3) Object identifiers are unique over all relations. (4) Rollbacks of transactions are considered as regular requests issued by clients. Extending Oshiya to schedule complex queries like joins or range queries is an interesting avenue for future work. Assumptions 2-4 simplify the presentation, but can be changed with minor modifications to Oshiya.

Scheduling queries and protocol specifications are given as domain relational calculus expressions. Capital letters denote variables, small letters indicate constants and $\epsilon$ denotes null. All variables not used in a universal quantification are implicitly existentially quantified. E.g., instead of $\exists A, B:(I(A, B) \wedge$ $\neg \exists C:(J(C, A)))$ we write $I(A, B) \wedge \neg J(C, A)$. Unrestricted existentially quantified variables are displayed as an underline ("-"), disjunctive use of constants by "". E.g., for the domain relational calculus expression $I(A, B) \wedge(A=a \vee A=c)$ we use the shortcut $I\left(a \mid c,_{-}\right)$. We define aggregation as: $\left\{G, F_{1}\left(A_{1}\right), \ldots, F_{n}\left(A_{n}\right) \mid\right.$ $E\}$. E is a domain relational calculus expression, $G$ is a set of attributes on which to group on (can be empty), and each $F_{i}$ is an aggregate over attribute $A_{i}$.

\section{Modeling Data Relation Snapshots and Defining the Oshiya Scheduling Relation Schemata for DSSI}

In order to implement DSSI with Oshiya, we have to (1) specify the schema of the scheduling relations which model the scheduler state, (2) formalize the protocol specification based on these relations (Sec. 5), and (3) implement the protocol specification as scheduling queries (Sec. 6). In this section, we show how to adapt data relation schemata to support data item versions (Sec. 4.1) and develop protocol-specific schemata for the Oshiya scheduling relations (Sec. 4.2).

\subsection{Modeling Snapshots with Data Relations}

We model snapshots explicitly by extending the schemata of data relations. This allows us to achieve DB independence and to run DSSI on DBMSs that do not support snapshots. We identify a version of data item $x$ using a tuple $(T A, S e q)$ where $T A$ is the transaction that created the version and $S e q$ is the position of the request within this transaction. Of course, versions can be modeled differently but this is orthogonal to our approach and beyond the scope of this paper. Given a database schema with relations $R_{1}, \ldots, R_{n}$, we map each relation $R_{i}$ to a relation $R_{i}^{\prime}$ which has four additional attributes. These attributes store the version identifier for the creator transaction (CTA and CSeq) and, if applicable, 
for the transaction that deleted the data item (DTA and DSeq). The primary key of $R_{i}^{\prime}$ is the primary key of $R_{i}$ union the attributes CTA and CSeq.

Example 6. Assume a bank stores account data with account numbers and balances in relation Accounts (AccNr,Bal). We map this relation to Accounts ${ }^{\prime}$ by extending its schema with the four additional attributes mentioned above. An example instance shown on the right contains an initial version of object $x$ created by transaction $t_{1}(C T A=1, C S e q=1)$ and two new versions created by $t_{2}$ and $t_{3}$.

Accounts'
\begin{tabular}{|l|l|l|l|l|l|}
\hline AccNr & Bal & CTA & CSeq & DTA & DSeq \\
\hline$x$ & 5 & 1 & 1 & - & - \\
\hline$x$ & 10 & 2 & 2 & - & - \\
\hline$x$ & 15 & 3 & 1 & - & - \\
\hline
\end{tabular}

\subsection{Oshiya Scheduling Relation Schemata}

For DSSI, we use the schemata for scheduling relations $\mathcal{R}, \mathcal{H}$ and $\mathcal{E}$ shown below. For simplicity, we present only attributes needed for scheduling and omit those necessary for request execution (e.g., the value to be written for write requests).

$\mathcal{R}$ (TA,Seq,Op,OID) $\quad \mathcal{H}$ (ID,TA,Seq,Op,OID,OTA,OSeq) $\quad \mathcal{E}$ (ID,TA,Seq,Op,OID,OTA,OSeq)

For each incoming request, we insert a tuple into $\mathcal{R}$ storing an identifier $T_{i}$ for the transaction $t_{i}$ that issued the request $(T A)$, the request position within this transaction $(S e q)$, the type of operation (read, write, abort or commit, stored in attribute $O p$ ) and the data object the requests is applied to $(O I D)$. Transactions identifiers $(T A)$ are ordered, i.e., if $b^{\circ} t_{i}<b o t_{j}$ then $T_{i}<T_{j}$. $\mathcal{H}$ and $\mathcal{E}$ contain additional attributes: $I D$ records the execution order of requests. For read requests, OTA and $O S e q$ store which object version was read by the request. These attributes correspond to the data relations attributes CTA and CSeq.

Example 7. Assume the instances of relations $\mathcal{R}$ and $\mathcal{H}$ displayed below. $\mathcal{H}$ contains the requests that produced the state of relation Accounts' from Ex. 6: (1) and (2) Transaction $t_{1}$ created the initial version of object $x$ and committed. (3) Transaction $t_{2}$ read this version of object $x$. (4) and (5) $t_{2}$ and $t_{3}$ wrote new versions of object $x$. (6) $t_{2}$ committed. (7) $t_{4}$ read the new version created by $t_{2}$. At this iteration, $\mathcal{R}$ contains no pending requests that have to be scheduled.

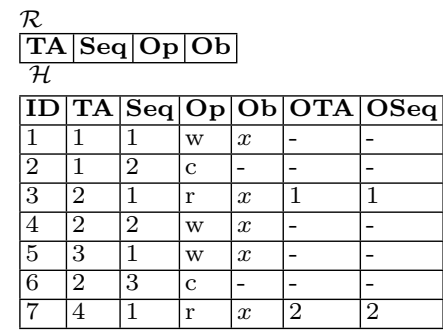

\section{DSSI Protocol Specification}

We now develop the protocol specification for DSSI based on the scheduling relations presented in Sec. 4. Recall from Sec. 3 that a protocol specification models a protocol as a set of domain relational calculus expressions over histories.

To formalize SI with Oshiya, we use views over relation $\mathcal{H}$ to get the relevant information described in Sec. 2. For bot, we use view $B O T(T A, I D)$ querying for each transaction (TA) the ID of its first request in $\mathcal{H}$. EOT $(T A, O p, I D)$ selects for each finished transaction $t_{i}(T A)$ the $I D$ of its final request in $\mathcal{H}$ (corresponds to eot ${ }_{i}$ ) and whether $t_{i}$ aborted or committed (Op). Overlap (TA1,TA2) 
contains all pairs of concurrently executed, non-aborted transactions, i.e., they do not have to be committed. PotPivotStr $(T A 1, T A 2, T A 3)$ selects all triples of transactions forming potential pivot structures as described in Sec. 2.3.

C1 (Read Versions) The SI protocol specifies $[3,5,14]$ that a read request $r_{i}(x)$ of a transaction $t_{i}$ reads $t_{i}$ 's most recent changes to $x$. If no such changes exist, then $r_{i}(x)$ reads the latest version of $x$ created by transactions that committed before $t_{i}$ started. These conditions are formalized as protocol specification constraint C1 (a) and (b) shown in Fig. 3: (a) The first case applies if a transaction $T$ has written object $O$ before reading a version $(X, Y)$ of $O$ :

$$
\mathcal{H}(I, T, N, r, O, X, Y) \wedge \mathcal{H}\left(I_{2}, T, N_{2}, w, O,_{-},{ }_{-}\right) \wedge I_{2}<I
$$

It follows that $T$ read a version it created itself $(X=T)$ and $(X, Y)$ is the latest version produced by $T$ before the read (no newer versions exist):

$$
X=T \wedge N_{2}=Y \wedge \neg\left(\mathcal{H}\left({ }_{-}, T, N_{2}, w, O_{,_{-},-}\right) \wedge Y<N_{2}<N\right)
$$

(b) The second case applies if $T$ has not written $O$ before the read was executed: $\neg\left(\mathcal{H}\left(I_{2}, T,_{-}, w, O,_{-},{ }_{-}\right) \wedge I_{2}<I\right)$. It follows that (1) $O$ was written by another transaction $X$ and $X$ committed before $T$ started. (2) $(X, Y)$ has to be the latest version written by $X$ and (3) there may not be another version written by a transaction $T_{2}$ that committed after $X$ but before $T$ started:
(1) $X \neq T \wedge \operatorname{EOT}\left(X, c, I_{3}\right) \wedge B O T\left(T, I_{4}\right) \wedge I_{3}<I_{4}$
(3) $\neg\left(\mathcal{H}\left({ }_{-}, T_{2},{ }_{-}, w, O,_{-},{ }_{-}\right) \wedge \operatorname{EOT}\left(T_{2}, c, I_{5}\right) \wedge I_{4}<I_{5}<I_{3}\right)$
(2) $\neg\left(\mathcal{H}\left({ }_{-}, X, N_{3}, w, O,_{-},-\right) \wedge N_{3}>Y\right)$

C2 (FCW) SI requires disjoint write-sets for all committed concurrent transactions. Protocol specification constraint C2 (see Fig. 3) models this condition as follows. If (1) two overlapping transactions $T$ and $T_{2}(2)$ both wrote the same object $O$ and (3) $T$ did already commit, then (4) $T_{2}$ did not commit:
(1) $\operatorname{Overlap}\left(T, T_{2}\right)$
(2) $\mathcal{H}\left({ }_{-}, T,_{-}, w, O,_{-},-\right) \wedge \mathcal{H}\left({ }_{-}, T_{2},{ }_{-}, w, O,_{-},-\right)$
(3) $\operatorname{EOT}\left(T, c,{ }_{-}\right)$
(4) $\neg E O T\left(T_{2}, c,-\right)$

C3 (Serializability) Recall that an SI history is serializable, if it does not contain pivot structures. In constraint C3 (see Fig. 3), we follow the approach outlined in Sec. 2.3: If (1) relation $\mathcal{H}$ contains a potential pivot structure, then we require that (2) at least one of the participating transactions did not commit:
(1) PotPivotStr $\left(T, T_{2}, T_{3}\right)$
(2) $\neg\left(E O T\left(T, c,{ }_{-}\right) \wedge E O T\left(T_{2}, c,{ }_{-}\right) \wedge E O T\left(T_{3}, c,{ }_{-}\right)\right)$

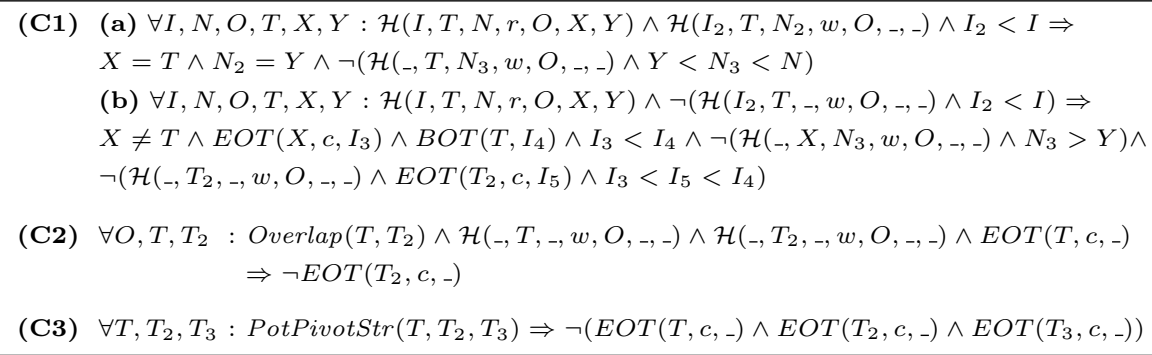

Fig. 3. DSSI Protocol Specification 


\section{DSSI Implementation}

Recall that with Oshiya, protocols are implemented as scheduling queries. We implemented all scheduling queries for DSSI, but in this paper we only describe $Q_{\text {Schedule }}$ Our prototype implementation of Oshiya requires the scheduling queries to be expressed in SQL. However, for conciseness, domain relational calculus expressions are used throughout this section. $Q_{\text {Schedule }}$ is developed in two steps. First we present queries necessary to detect potential pivot structures (Sec. 6.1). Afterwards, we use these queries to implement $Q_{\text {Schedule }}$ (Sec. 6.2). Recall that detecting potential pivot structures and aborting one of the participating transactions ensure serializability. However, this approach may detect false positives (see Sec. 2). Studying the trade-off between the number of false positives and the cost of scheduling is an interesting avenue for future work.

\subsection{Detecting Potential Pivot Structures}

We now discuss how to express BOT, EOT, Overlap and PotPivotStr introduced in Sec. 5 as queries over $\mathcal{H}$. BOT and EOT are defined below. E.g., EOT queries for each finished transaction $T$ its abort resp. commit state $(A)$ and its eot $(I)$ which is equal to the $I D$ of its abort resp. commit request in $\mathcal{H}$.

$$
\begin{aligned}
& B O T=\left\{T, I \mid \mathcal{H}\left(I, T,_{-,-},,_{-},-\right) \wedge \neg\left(\mathcal{H}\left(I_{2}, T_{,_{-},,_{-},-,}\right) \wedge I_{2}<I\right)\right\} \\
& E O T=\left\{T, A, I\left|\mathcal{H}\left(I, T_{-}, A,_{-},-,-\right) \wedge A=a\right| c\right\}
\end{aligned}
$$

Overlapping transactions are inferred as specified below. Two (1) non-aborted transactions $T_{1}$ and $T_{2}$ overlap if (2) bot $t_{1}<^{H}$ bot $_{2}$ and (3) bot ${ }_{2}<^{H} c_{1}$ (if $T_{1}$ has already committed) or (4) the symmetric case holds:

$$
\begin{aligned}
\text { Overlap }= & \left\{T_{1}, T_{2} \mid T_{1} \neq T_{2} \wedge \neg E O T\left(T_{1} \mid T_{2}, a,-\right) \wedge\right. \\
& \left(\left(B O T\left(T_{1}, I\right) \wedge B O T\left(T_{2}, I_{2}\right) \wedge I<I_{2} \wedge\right.\right. \\
& \left.\left(E O T\left(T_{1}, c, I_{3}\right) \Rightarrow I_{2}<I_{3}\right)\right) \vee \\
& \left.\left.\left(B O T\left(T_{2}, I_{2}\right) \wedge B O T\left(T_{1}, I\right) \wedge I_{2}<I \wedge\left(\operatorname{EOT}\left(T_{2}, c, I_{3}\right)=>I<I_{3}\right)\right)\right)\right\}
\end{aligned}
$$

We use PotVulnEdge to query all potential vulnerable edges between concurrent, non-aborted transactions $T$ and $T_{2}$ (potential, because $T$ and $T_{2}$ might not yet have committed). PotPivotStr detects potential pivot structures by checking for transactions $\left(T_{2}\right)$ that have both an incoming and outgoing PotVulnEdge:

$$
\begin{aligned}
\text { PotVulnEdge } & =\left\{T, T_{2} \mid \mathcal{H}\left(I, T,_{-}, r, O,_{-},{ }_{-}\right) \wedge \mathcal{H}\left(I_{2}, T_{2},-, w, O,_{-},-\right) \text {Overlap }\left(T, T_{2}\right) \wedge I<I_{2}\right\} \\
\text { PotPivotStr } & =\left\{T, T_{2}, T_{3} \mid \operatorname{PotVulnEdge}\left(T, T_{2}\right) \wedge \operatorname{PotVulnEdge}\left(T_{2}, T_{3}\right)\right\}
\end{aligned}
$$

Example 8. We show the results of the queries defined above (highlighted) for the history state $\mathcal{H}$ from Ex. 7. For instance, PotVulnEdge contains one potential vulnerable edge from transaction $t_{2}$ to $t_{3}$, because $t_{2}$ and $t_{3}$ overlap and $t_{2}$ read object $x$ and afterwards $t_{3}$ wrote a new version of object $x\left(r_{2}(x)<^{H} w_{3}(x)\right)$.
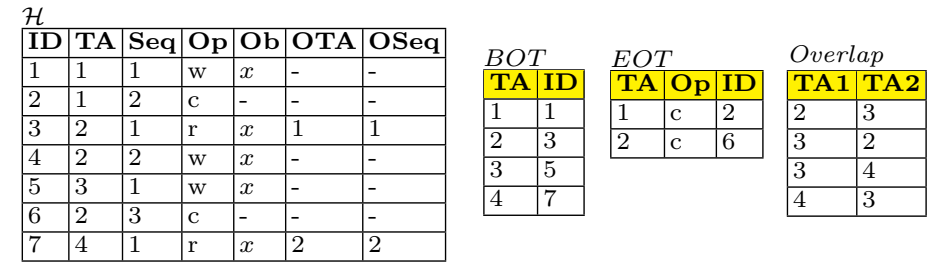

PotVulnEdge

TAout TAin

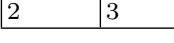

PotPivotStr

\begin{tabular}{|l|l|l|l|}
\hline TA1 & TA2 & TA3 \\
\hline
\end{tabular} 


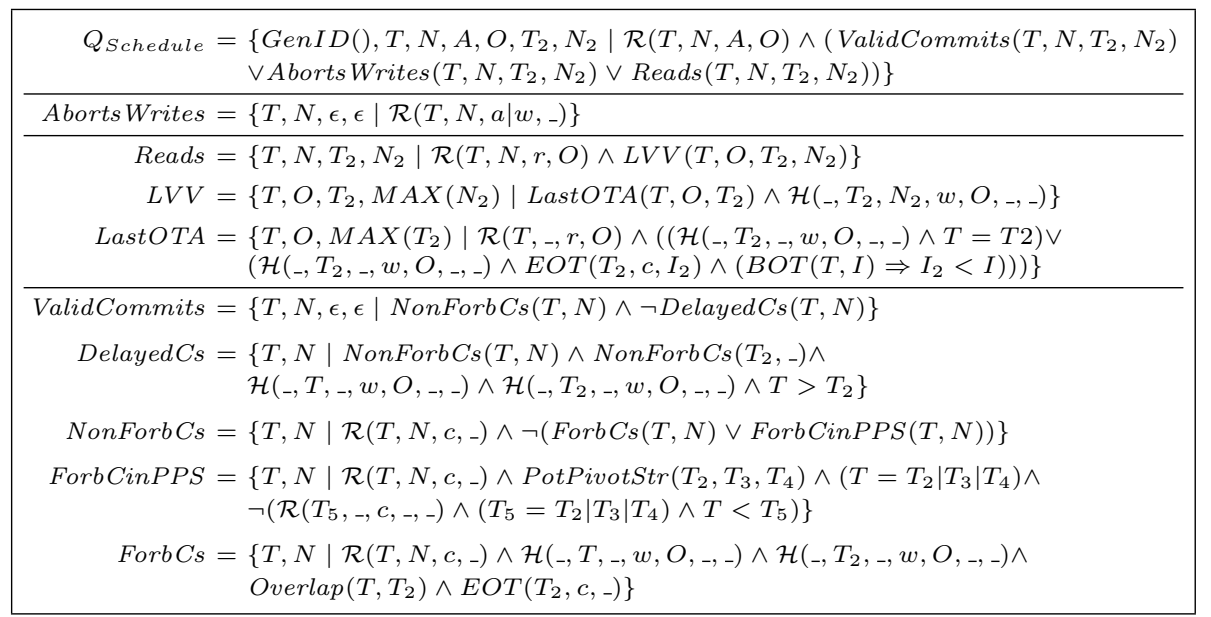

Fig. 4. $Q_{\text {Schedule }}$

\section{2 $Q_{\text {Schedule }}$}

The DSSI version of $Q_{\text {Schedule }}$ implementing the protocol specification constraints C1-C3 is shown in Fig. 4. According to the SI conditions, all write, abort, and read requests from $\mathcal{R}$ may always be selected for execution. $Q_{\text {Schedule }}$ selects all of these requests using queries AbortWrites and Reads. Which commit requests can be selected without violating constraints $C 2$ and $C 3$ is determined through query ValidCommits. In $Q_{\text {Schedule }}$, function $\operatorname{GenID()}$ generates unique values for the $I D$ attribute of $\mathcal{H}$ (modelling the execution order of requests).

Read Requests (C1) The Reads query uses $L V V$ (last valid version) to select for each read request of transaction $T$ on object $O$ the version $\left(T_{2}, N_{2}\right)$ that has to be read. Recall that attributes $O T A$ and $O S e q$ of relations $\mathcal{E}$ and $\mathcal{H}$ identify a version of an object $O$. Version $\left(T_{2}, N_{2}\right)$ is computed in two steps. LastOTA queries the transaction identifier $\left(T_{2}\right)$ of the transaction that wrote the version of $O$ that has to be read by $T$. Based on this information $L V V$ determines $N_{2}$, the $S e q$ value of the latest write request of $T_{2}$ on object $O . T_{2}$ is the maximal value from the following union: (a) $T_{2}=T$ if $T$ itself created versions of $O$ and (b) transactions that wrote a version of $O$ and committed before $T$ started.

(a) $\mathcal{H}\left({ }_{-}, T_{2},{ }_{-}, w, O,_{-},{ }_{-}\right) \wedge T=T_{2} \quad(\mathbf{b}) \mathcal{H}\left({ }_{-}, T_{2},{ }_{-}, w, O,_{-},{ }_{-}\right) \wedge E O T\left(T_{2}, c, I_{2}\right) \wedge\left(B O T(T, I) \Rightarrow I_{2}<I\right)$

Example 9. Consider $\mathcal{H}$ from Ex. 8. $r_{2}(x)$ read the initial version of object $x$ (since $c_{1}<{ }^{H} b_{2}$ ) and $r_{4}(x)$ read the version written by $t_{2}$ (since $c_{2}<^{H}$ bot $_{4}$ ).

Commit Requests (C2 and C3) To guarantee that constraints C2 and C3 hold for each history produced by $Q_{S c h e d u l e}$, we have to prevent commit requests to be executed if (1) the commit would violate the FCW rule $(\mathrm{C} 2)$ or $(2)$ the commit would violate serializability (C3). There are two possible ways how the execution of commit requests can violate the FCW rule: (1a) A commit is from a transaction whose write-set overlaps with the one of a concurrent but already committed transaction and (1b) if $\mathcal{R}$ contains commit requests from multiple 
transactions with overlapping write-sets, then only one of these transaction may commit. Note that in the concrete implementation, commits identified to violate C2 or C3 are selected by $Q_{\text {Revoked }}$ and aborted.

We use a two stage approach to select valid commits: In step 1, query NonForbCs selects commits from $\mathcal{R}$ and filters out commits of case 1a using query ForbCs and those of case 2 using query ForbCinPPS. NonForbCs may still contain sets of commit requests from transactions with overlapping write-sets (case 1b). We only allow the oldest transaction from each set to commit. Therefore, in step 2, query ValidCommits selects all requests from NonForbCs and uses query DelayedCs to keep only the commit request of the oldest transaction for each set of transactions with overlapping write-sets.

Step 1 Query ForbCs (case 1a) identifies commits of transactions $T$ that (a) wrote an object also written by an (b) overlapping committed transaction $T_{2}$.
(a) $\mathcal{H}\left({ }_{-}, T,_{-}, w, O,_{-},-\right) \wedge \mathcal{H}\left({ }_{-}, T_{2},-, w, O,_{-},-\right)$
(b) $\operatorname{Overlap}\left(T, T_{2}\right) \wedge \operatorname{EOT}\left(T_{2}, c,,_{-}\right)$

ForbCinPPS (case 2) selects a commit of transaction $T$ from $\mathcal{R}$ if (a) $T$ belongs to potential pivot structure $p$ and (b) $\mathcal{R}$ does not contain a commit request of a younger transaction $T_{5}$ (recall that bot $_{1}<$ bot $_{2} \Rightarrow T_{1}<T_{2}$ ) also belonging to $p$. Thus, if $\mathcal{R}$ contains commits of more than one of the transactions belonging to $p$, we disallow only the youngest one to commit (and abort it using $Q_{\text {Revoked }}$ ).

(a) PotPivotStr $\left(T_{2}, T_{3}, T_{4}\right) \wedge\left(T=T_{2}\left|T_{3}\right| T_{4}\right) \quad(\mathbf{b}) \neg\left(\mathcal{R}\left(T_{5},,_{-}, c_{-},,_{-}\right) \wedge\left(T_{5}=T_{2}\left|T_{3}\right| T_{4}\right) \wedge T<T_{5}\right)$

Example 10. Consider the instances of $\mathcal{R}$ and $\mathcal{H}$ shown below that model history $H_{w s}$ from Fig. 1. To keep the example simple, we do not show the actions of transaction $t_{0}$ that created the initial versions of objects $x$ and $y$. Requests $c_{1}$ and $c_{2}$ belong to the same potential pivot structure $p$. Their execution can lead to a write skew violating C3. $Q_{S \text { chedule }}$ selects $c_{1}$ (smallest $T A$ value). $c_{2}$ (commit of youngest transaction) is selected by ForbCinPPS and aborted to break $p$.
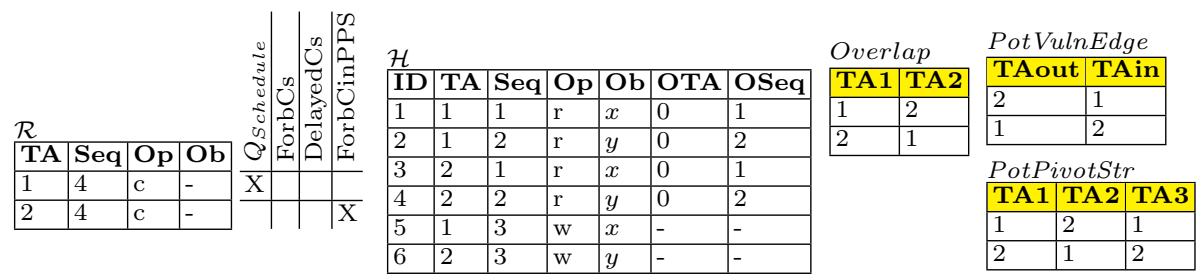

Step 2 DelayedCs detects case 1b by selecting all transactions $T$ from NonFor$b C s$ where (a) NonForbCs contains another transaction $T_{2}$ which (b) wrote an object $O$ that has also been written by $T$ and (c) which is older than $T$.
(a) $\operatorname{NonForbCs}\left(T_{2},-\right)$
(b) $\mathcal{H}\left({ }_{-}, T,,_{-}, w, O,_{-},-\right) \wedge \mathcal{H}\left({ }_{-}, T_{2},{ }_{-}, w, O,_{-},-\right)$
(c) $T>T_{2}$

Example 11. Consider the instances of $\mathcal{R}$ and $\mathcal{H}$ displayed below. $Q_{\text {Schedule }}$ selects all read $\left(r_{6}(x)\right)$ and write $\left(w_{7}(y)\right)$ requests. $c_{3}$ belongs to ForbCs because transaction $t_{3}$ wrote the same object as the concurrent but already committed transaction $t_{2}$ and is, thus, not allowed to commit. $c_{4}$ and $c_{5}$ belong to NonFor$b C s$, but $t_{4}$ and $t_{5}$ both wrote the same object $x$. ValidCommits selects only $c_{4}$ (oldest transaction from the set $\left\{t_{4}, t_{5}\right\}$ of transactions with overlapping writeset). $c_{5}$ is filtered out by DelayedCs. 

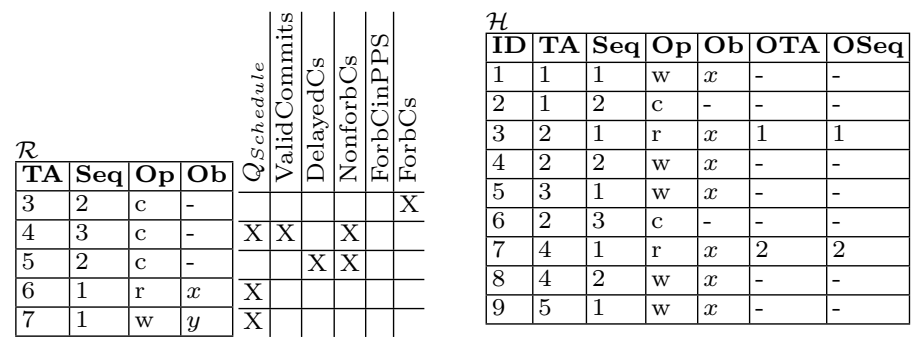

\section{Correctness Analysis}

We now proof that every history produced under DSSI is serializable. Recall that an SI history is serializable if it does not contain a pivot structure. Thus, we can show this fact by proving that $\mathcal{H}$ cannot contain a potential pivot structure between committed transactions (equivalent after Sec. 2.3). Note that the influence of the other scheduling queries (mentioned in Sec. 3) on the results of $Q_{\text {Schedule }}$ and the compliance of $\mathrm{C} 1$ and $\mathrm{C} 2$ are not in the scope of this paper.

Theorem 1 ( Q Schedule Prevents Pivot Structures). $Q_{\text {Schedule }} \models C 3$

Proof. We omit to prove that the query PotPivotStr returns all potential pivot structures contained in $\mathcal{H}$, because the proof is trivial. We proof Theorem 1 by contradiction. Assume the negation of C3 holds:

$$
\begin{aligned}
& \neg\left(\forall T, T_{2}, T_{3}: \text { PotPivotStr }\left(T, T_{2}, T_{3}\right) \Rightarrow \neg\left(\operatorname{EOT}\left(T, c,{ }_{-}\right) \wedge \operatorname{EOT}\left(T_{2}, c,_{-}\right) \wedge \operatorname{EOT}\left(T_{3},,_{,},_{-}\right)\right)\right. \\
\Leftrightarrow \quad \exists T, T_{2}, T_{3}: & \text { PotPivotStr }\left(T, T_{2}, T_{3}\right) \wedge \operatorname{EOT}\left(T, c,_{-}\right) \wedge \operatorname{EOT}\left(T_{2}, c,,_{-}\right) \wedge \operatorname{EOT}\left(T_{3}, c,_{-}\right)
\end{aligned}
$$

Let $k$ be the first scheduler iteration where this equation holds for a fixed $T_{1}$, $T_{2}, T_{3}$ and $T_{4}$.

$\Leftrightarrow \exists T, T_{2}, T_{3}, k: \operatorname{PotPivotStr}_{k}\left(T, T_{2}, T_{3}\right) \wedge \operatorname{EOT}_{k}\left(T, c,_{-}\right) \wedge \operatorname{EOT}_{k}\left(T_{2}, c,_{-}\right) \wedge E_{k}\left(T_{3}, c,_{-}\right)$

Without loss of generality, let $T_{3}$, the transaction at the third position of the potential pivot structure (PotPivotStr $\left.\left(T, T_{2}, T_{3}\right)\right)$, be the youngest transaction of the participating transactions. This assumption does not result in a loss of generality, because the position of $T_{3}$ is irrelevant for the rest of the proof. There must exist a scheduler iteration $i<k$ where $T_{3}$ has not yet committed but already belongs to PotPivotStr.

$$
\Rightarrow \exists i: \operatorname{PotPivotStr}_{i}\left(T, T_{2}, T_{3}\right) \wedge T_{3}>T \wedge T_{3}>T_{2} \wedge \neg E O T_{i}\left(T_{3}, c,-\right)
$$

It follows that the commit request $c_{3}$ of $T_{3}$ occurs in relation $\mathcal{R}$ at some scheduler iteration $j(i<j<k)$. To be executed, $c_{3}$ has to belong to the set of non-forbidden commits (NonForbCs). We can assume $\operatorname{PotPivotStr}_{i}\left(T, T_{2}, T_{3}\right) \Rightarrow$ $\operatorname{PotPivotStr}_{j}\left(T, T_{2}, T_{3}\right)$.

$$
\Rightarrow \exists j: \operatorname{PotPivotStr}_{j}\left(T, T_{2}, T_{3}\right) \wedge T_{3}>T \wedge T_{3}>T_{2} \wedge \neg E O T_{j}\left(T_{3}, c,{ }_{-}\right) \wedge \operatorname{NonForbCs}_{j}\left(T_{3},{ }_{-}\right)
$$

We now replace NonForbCs by its definition and, afterwards, remove terms that are not needed to derive the contradiction:

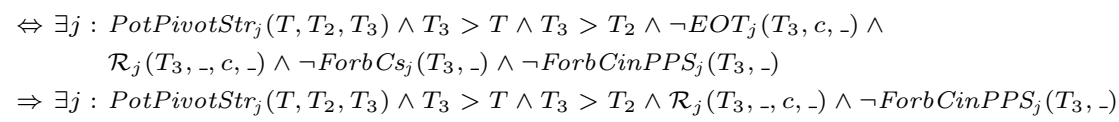


Since $c_{3}$ in $\mathcal{R}$ is the commit request of the youngest transaction participating in $p, \mathcal{R}$ cannot contain a commit request of a transaction that is both younger than $T_{3}$ and also belongs to $p$ :

$$
\begin{aligned}
\Leftrightarrow \exists j: & \mathcal{R}_{j}\left(T_{3},{ }_{-}, c,{ }_{-}\right) \wedge \text { PotPivotStr } \\
& \rightarrow\left(T, T_{2}, T_{3}\right) \wedge \neg\left(\mathcal{R}_{j}\left(T_{4},_{-}, c_{-},_{-}\right) \wedge T_{4}=T \mid T_{2} \wedge T_{4}<T_{3}\right) \wedge \\
& \neg \text { ForbCinPPS } S_{j}\left(T_{3},{ }_{-}\right)
\end{aligned}
$$

From the first line of the equation shown above, we can follow $\operatorname{Forb} \operatorname{CinPP} S_{j}\left(T_{3},{ }_{-}\right)$ which leads to the contradiction and, thus, proves Theorem 1:

$$
\Rightarrow \exists j: \operatorname{ForbCinPP} S_{j}\left(T_{3},{ }_{-}\right) \wedge \neg F \operatorname{ForbCinPP} S_{j}\left(T_{3},-\right) \Rightarrow \underline{\not}
$$

\section{Related Work}

The ACTA framework allows to formalize properties of transaction models using first-order formulas over schedules [6]. Its conciseness and clarity inspired us to implement schedulers based on declarative protocol specifications. The basic ideas of Oshiya have been presented in [13], but this work focused on single-version protocols (2PL) and did not consider correctness. Recent research projects leverage the advantages of declarative languages in various areas $[2,4$, $7,12,15,16]$. The Boom approach uses Overlog to build distributed systems [2], e.g., a scheduler for MapReduce tasks with policies like First-Come-First-Served. In contrast to our approach, Boom does not focus on DB requests or consistency.

Application analysis techniques have been presented in $[10,9]$ to determine if applications generate serializable executions when running on a system that applies SI. The key idea is that DBAs analyze transaction programs, produce static dependency graphs and manually check for dangerous access patterns leading to non-serializability. Some approaches modify transaction programs to ensure serializable SI schedules: Fekete [9] proposed the techniques Materialize and Promotion to achieve serializability. Jorwekar et al. [11] tried to automate the check whether non-serializable SI executions can occur. However, this approach still requires manual confirmation and modification. Fekete [8] executes certain transactions of pivot structures under S2PL, others run under SI. This approach requires the underlying platform to support both S2PL and SI. Alomari et al. [1] set exclusive locks in an External Lock Manager (ELM) to ensure serializability with SI. In contrast to DSSI, these approaches do not work for ad-hoc transactions and require static analysis or manual program modifications.

Another line of work focused on modifying the SI algorithm of the underlying system to ensure serializability. The closest approach to DSSI is the SSI protocol [5] described in Sec. 2.3. This approach modifies the DB lock manager with an additional type of locks that are used to detect potential pivot structures. DSSI infers all necessary information to detect and prevent these structures from relation $\mathcal{H}$. Our implementation works with DBMSs out of the box. The underlying DBMS does not even need to provide SI since we model data versions in a standard relational schema (see Sec. 4). Using Oshiya, the implementation of DSSI is close to its formal specification, which enabled us to prove its correctness. 


\section{Conclusions and Future Work}

We develop Declarative Serializable Snapshot Isolation (DSSI) using our declarative scheduling model Oshiya. DSSI ensures serializable schedules by avoiding pivot structures and provides DB independence. We formally define DSSI as a Oshiya protocol specification, present a scheduler implementation, and prove that the implementation ensures serializability. In future work, we will experimentally evaluate the performance of DSSI and investigate the trade-offs involved in reducing the amount of false positives.

\section{References}

1. M. Alomari, A. Fekete, and U. Röhm. A Robust Technique to Ensure Serializable Executions with Snapshot Isolation DBMS. In ICDE, pages 341-352, 2009.

2. P. Alvaro, T. Condie, N. Conway, K. Elmeleegy, J. M. Hellerstein, and R. Sears. Boom Analytics: Exploring Data-Centric, Declarative Programming for the Cloud. In EuroSys, pages 223-236, 2010.

3. H. Berenson, P. Bernstein, J. Gray, J. Melton, E. O'Neil, and P. O'Neil. A Critique of ANSI SQL Isolation Levels. In SIGMOD, pages 1-10, 1995.

4. A. Böhm, E. Marth, and C.-C. Kanne. The Demaq System: Declarative Development of Distributed Applications. In SIGMOD, pages 1311-1314, 2008.

5. M. J. Cahill, U. Röhm, and A. D. Fekete. Serializable Isolation for Snapshot Databases. TODS, 34(4):1-42, 2009.

6. P. K. Chrysanthis and K. Ramamritham. ACTA: A Framework for Specifying and Reasoning about Transaction Structure and Behavior. In SIGMOD, pages 194-203, 1990.

7. D. Chu, L. Popa, A. Tavakoli, J. M. Hellerstein, P. Levis, S. Shenker, and I. Stoica. The Design and Implementation of a Declarative Sensor Network System. In SenSys, pages 175-188, 2007.

8. A. Fekete. Allocating Isolation Levels to Transactions. In PODS, pages 206-215, 2005.

9. A. Fekete, D. Liarokapis, E. O'Neil, P. O'Neil, and D. Shasha. Making Snapshot Isolation Serializable. ACM Trans. Database Syst., 30(2):492-528, 2005.

10. A. D. Fekete. Serialisability and Snapshhot Isolation. In $A D C$, pages 201-209, 1999.

11. S. Jorwekar, A. Fekete, K. Ramamritham, and S. Sudarshan. Automating the Detection of Snapshot Isolation Anomalies. In VLDB, pages 1263-1274, 2007.

12. L. Kot, N. Gupta, S. Roy, J. Gehrke, and C. Koch. Beyond Isolation: Research Opportunities in Declarative Data-Driven Coordination. SIGMOD Rec., 39:27-32, 2010.

13. C. Tilgner. Declarative Scheduling in Highly Scalable Systems. In EDBT Ph.D. Workshop, 2010.

14. G. Weikum and G. Vossen. Transactional Information Systems. Morgan Kaufmann Publishers, 2002.

15. W. White, A. Demers, C. Koch, J. Gehrke, and R. Rajagopalan. Scaling Games to Epic Proportions. In SIGMOD, pages 31-42, 2007.

16. F. Yang, J. Shanmugasundaram, M. Riedewald, and J. Gehrke. Hilda: A HighLevel Language for Data-Driven Web Applications. In ICDE, 2006. 